\title{
Adrenal Fluid or Secretion
}

National Cancer Institute

\section{Source}

National Cancer Institute. Adrenal Fluid or Secretion. NCI Thesaurus. Code C13416.

Epinephrine and norepinephrine from the medulla and steroid hormones from the cortex. 